Effect of microencapsulation (maltodextrin-chinchayote starch) on the antioxidant capacity of the Capulín (Prunus serotina)

\title{
Efecto de la microencapsulación (maltodextrina-almidón de chinchayote) en la capacidad antioxidante del Capulín (Prunus serotina)
}

Elsa Díaz-Montes, Jesús Antonio Valencia-Arredondo, Jorge Yáñez-Fernández*

Laboratorio de Biotecnología Alimentaria, Unidad Profesional Interdisciplinaria de Biotecnología, Instituto Politécnico Nacional. Av. Acueducto S/N Col. Barrio La Laguna, Ticoman, Ciudad de México, CP 07340, México.

Tel.: (+52) 5557296000 Ext. 56477

*Corresponding author.

E-mail address: jyanezfe@ipn.mx (J. Yánez-Fernández).

Article history:

Received: 29 December 2017 / Received in revised form: 12 March 2018 / Accepted: 18 March 2018 / Published online: 1 April 2018.

https://doi.org/10.29267/mxjb.2018.3.2.23

\begin{abstract}
Capulín fruit (FC) contains bioactive compounds, mainly phenolic, which are valued by food and pharmaceutical industry for their antioxidant activity. However, environmental conditions causes polyphenolic compounds degradation and loss for low stability. Spraydrying as a microencapsulation method turned out to be a viable alternative for protection of polyphenols extracted from FC; evaluating 8 mixtures of maltodextrin (MD) and chinchayote starch (AC) as encapsulants (1-10\% concentration), T8 treatment (10-0\% MDAC) resulted in a powder with low water activity and it obtained the best performance and solubility results $(89.13$ and $75.43 \%$, respectively), in addition, treatment had good results in phenolic content (109.75 $\mathrm{mg} \mathrm{GAE} / \mathrm{mL})$ and antioxidant activity $(51.89 \mathrm{mg} \mathrm{AAE} / \mathrm{mL})$. Therefore, the Project proposes the modification of the solubility of the AC to re-evaluate it as an encapsulant agent.
\end{abstract}

Keywords: antioxidant capacity, Capulín fruit, maltodextrin-chinchayote starch, microencapsulation, spray-drying. 


\section{RESUMEN}

El fruto del Capulín (FC) contiene compuestos bioactivos, de carácter fenólico principalmente, que son valorados por la industria alimentaria y farmacéutica por la actividad antioxidante que poseen. Sin embargo, la poca estabilidad que tiene este tipo de compuestos ante condiciones medioambientales provoca su degradación y pérdida. El secado por aspersión como método de microencapsulamiento resulto ser una alternativa viable para la protección de los polifenoles extraídos del FC; probándose 8 mezclas de maltodextrina (MD) y almidón de chinchayote (AC) como agentes encapsulantes (1-10\% de concentración), el tratamiento T8 (10-0\% MD-AC) resulto en un polvo con poca actividad de agua y obtuvo los mejores resultados de rendimiento y solubilidad (89.13 y $75.43 \%$, respectivamente), además de presentar buenos resultados en cuando al contenido fenólico (109.75 mg GAE/mL) y a la actividad antioxidante (51.89 mg AAE/mL). Por tal, el proyecto propone la modificación de la solubilidad del AC para re-evaluarlo como encapsulante.

Palabras clave: maltodextrina-almidón de chinchayote, capacidad antioxidante, fruto del Capulín, microencapsulación, secado por aspersión.

\section{INTRODUCCIÓN}

\subsection{Fruto del Capulín}

El Capulín (Prunus serotina) es un árbol originario de América que habita climas cálidos, semicálidos, secos y templados, que desde tiempos antiguos se ha utilizado empíricamente como tratamiento para la tos, gripe, diarrea, dolor abdominal y fiebre (BDMTM, 2009). El árbol produce un fruto (FC) pequeño de color verde a rojo oscuro, que por su alto contenido en azúcares es empleado en la elaboración de jaleas, mermeladas y dulces (Ordaz-Galindo et al., 1999); sin embargo, el FC atrae la atención por su contenido en componentes bioactivos de carácter fenólico, que le confieren principalmente una actividad antioxidante (Ordaz-Galindo et al., 1999; Jiménez et al., 2011).

De acuerdo a su definición un compuesto bioactivo es todo constituyente que posee carácter nutricional, se encuentra en pequeñas cantidades en los alimentos y genera beneficios a la salud de quien lo consume (Pennington, 2002); estos compuestos son generados esencialmente por el metabolismo secundario de las plantas superiores por lo que los principales hacen referencia a los carotenoides, flavonoides, ácidos fenólicos, esteroles vegetales, proteínas y compuestos orgánicos (Belloso et al., 2009). Algunos estudios en el FC han revelado la presencia otro tipo de componentes de interés como son los ácidos grasos (oleico, linoleico y oleosteárico), terpenos, compuestos volátiles (2,3-butanodiol y benzaldehído) y algunos otros de naturaleza orgánica, que además de la propiedad antioxidante también presentan actividad antimicrobiana; sin embargo, la poca estabilidad que tiene este tipo de compuestos ante condiciones medioambientales provoca su degradación y pérdida (Biessesls et al., 1974; Jimenez et al., 2011; García-Aguilar et al., 2015). 


\subsection{Microencapsulación}

La encapsulación es un proceso físico en donde se generan pequeños aglomerados que atrapan componentes en una matriz polimérica (Deladino et al., 2008), ofreciéndoles una estabilidad y protección ante condiciones ambientales como la luz, la humedad y el oxígeno (Fang \& Bhandari, 2010). Este tipo de tecnologías han sido implementadas en la industria alimentaria para el atrapamiento de aminoácidos, vitaminas, minerales, colorantes, enzimas, edulcorantes y compuestos antioxidantes principalmente (Deladino et al., 2008). Por otro lado, el termino microencapsulación hace referencia al recubrimiento de sustancias de bajo peso molecular o de pequeñas cantidades de compuestos (Yáñez et al., 2002), por lo que se obtienen micro capsulas que generalmente son esféricas y semipermeables (Anal \& Singh, 2007).

Dentro del proceso de microencapsulación existen dos factores importantes a considerar, el primero se relaciona con la selección del agente encapsulante (Porras-Saavedra et al., 2015), pues de esto depende la estabilidad de los componentes y la eficiencia del proceso (Gharsallaoui et al., 2007), de tal manera que en el Tabla 1 se muestran los principales polímeros empleados en el proceso; el segundo factor tiene que ver con el método de encapsulación que se requiera, pues este dependerá del tipo de componentes bioactivos que se desea proteger, por lo que la Tabla 2 muestra los métodos más utilizados en la encapsulación (Desai \& Park, 2005).

Tabla 1. Principales materiales de encapsulación de aditivos alimentarios.

\begin{tabular}{cc}
\hline Tipo de cubierta & Material encapsulante \\
\hline & Almidón \\
Maltodextrina & Quitosano \\
Carbohidratos & Sólidos de jarabe de maíz \\
& Dextrano \\
& Almidón modificado \\
& Ciclodextrina \\
\hline Celulosa & Carboximetilcelulosa \\
& Metil celulosa \\
& Etil celulosa \\
& Acetato ftalato de celulosa \\
& Acetato de butirato-ftalato de celulosa \\
\hline Gomas & Goma acacia \\
& Agar \\
& Alginato de sodio \\
Carragenina \\
\hline \multirow{2}{*}{ Lípidos } & Cera \\
& Parafina \\
& Cera de abeja \\
\hline
\end{tabular}




\begin{tabular}{cc}
\hline & Diacil-gliceroles \\
Aceites \\
Grasas \\
\hline \multirow{3}{*}{ Proteínas } & Gluten \\
& Caseína \\
& Gelatina \\
Albumina \\
Péptidos \\
\hline
\end{tabular}

Adaptada de Desai \& Park, 2005

Tabla 2. Principales técnicas de microencapsulación.

\begin{tabular}{ccc}
\hline Tipo de proceso & Método & Componente bioactivo \\
\hline \multirow{3}{*}{ Físicos } & Aceite de naranja \\
& Secado por aspersión & Acetato de linalol \\
& Secado por enfriamiento & Cardamomo \\
& Secado por congelamiento & Limoneno \\
& Extrusión por centrifugación & Sabores \\
& Liofilización & Vitaminas \\
& & Colorantes \\
\hline \multirow{2}{*}{ Químicos } & Linalol \\
& Lecho fluidizado & Aceite de cáscara de naranja \\
& Por liposomas & d-Limoneno \\
& Inclusión compleja & Aceite de limón \\
& & Sabor café natural \\
& & Sabor café sintético \\
Fisicoquímicos & Sólidos \\
& & Productos farmacéuticos \\
\hline & Coacervación & Aceite de romero \\
& Cocristalización & Aceite de menta \\
& & Teobromina \\
& & Aditivos alimentarios \\
\hline
\end{tabular}

El proceso de secado por aspersión es uno de los métodos de microencapsulación más utilizados a nivel industrial, pues al ser un método físico no requiere demasiados consumibles y los resultados son polvos con una mayor vida de anaquel (Shahidi \& Han, 1993). El funcionamiento de un secador por aspersión se basa en la atomización de una dispersión total o casi totalmente liquida (que contiene el compuesto activo y el agente encapsulante) a través de un gas caliente que quita la humedad y permite que el agente encapsulante atrape al compuesto (Phisut, 2012). 
De tal manera, el empleo de operaciones unitarias como el secado por aspersión, representan una alternativa útil para la preservación de compuestos, por su alto rendimiento, bajo costo y por ser una herramienta confiable para mantener el carácter bioactivo de dichos compuestos.

\section{MATERIALES Y MÉTODOS}

\subsection{Materiales}

\subsubsection{Reactivos químicos}

Ácido ascórbico $\left(\mathrm{C}_{6} \mathrm{H}_{8} \mathrm{O}_{6}\right)$, ácido gálico $\left(\mathrm{C}_{7} \mathrm{H}_{6} \mathrm{O}_{5}\right)$, ácido sulfúrico $\left(\mathrm{H}_{2} \mathrm{SO}_{4}\right)$, agua $\left(\mathrm{H}_{2} \mathrm{O}\right)$, carbonato sódico $\left(\mathrm{Na}_{2} \mathrm{CO}_{3}\right)$, DPPH $\bullet$ (1,1-difenil-2-picrilhidrazil), etanol $\left(\mathrm{C}_{2} \mathrm{H}_{5} \mathrm{OH}\right)$, éter etílico $\left(\mathrm{C}_{4} \mathrm{H}_{10} \mathrm{O}\right)$, fenolftaleína $\left(\mathrm{C}_{20} \mathrm{H}_{14} \mathrm{O}_{4}\right)$, fenol $\left(\mathrm{C}_{6} \mathrm{H}_{6} \mathrm{O}\right)$, glucosa $\left(\mathrm{C}_{6} \mathrm{H}_{12} \mathrm{O}_{6}\right)$, hidróxido de sodio $(\mathrm{NaOH})$, maltodextrina $10-\mathrm{DE}\left(\mathrm{C}_{60} \mathrm{H}_{102} \mathrm{O}_{51}\right)$, reactivo de Folin-Ciocalteu y Trolox (ácido 6-hidroxi-2,5,7,8-tetrametilcloro-2-carboxilico); fueron de grado analítico obtenidos de Sigma-Aldrich (Toluca, México).

\subsubsection{Materiales y equipos}

Centrifuga (IEC HN-SII), estufa de secado (HCM-A45, TECNI LAB), higrómetro (3TE, Aqua Lab), malla (\#200), microscopio electrónico de barrido (JSM 7800F, JEOL), papel filtro (Whatman \#1), pH-metro portátil (pHep, HANNA), refractómetro análogo portátil (FG-109, Zhifong), secador por aspersión (B-2090, BÜCHI) y vortex (G560, SCIENTIFIC INDUSTRIES); proporcionados por el laboratorio de Biotecnología Alimentaria de la UPIBI-IPN.

\subsubsection{Material biológico}

El extracto de Capulín (EC) se obtuvo macerado y mezclado el fruto (región de la Meseta Tarasca) con agua $(1: 5, \mathrm{w} / \mathrm{v})$ como solvente de extracción, el cual posteriormente se centrifugó (3000 rpm, $5 \mathrm{~min}$ ) y se filtró al vacío; el filtrado se congeló a $-60^{\circ} \mathrm{C}$ en oscuridad hasta su uso. El almidón de chinchayote (AC) se extrajo (modificado de Ganga \& Corke, 1999) triturando el tubérculo (región de la Meseta Tarasca) con agua $(1: 1 \mathrm{w} / \mathrm{v})$ y el residuo acuoso se filtró, la torta formada se re-trituró con agua $(1: 4 \mathrm{w} / \mathrm{v})$ y el extracto se filtró nuevamente; una vez obtenido el filtrado, este se lavó 4 veces con agua $(1: 4 \mathrm{w} / \mathrm{v})$ y se dejó sedimentar para posteriormente retirar el agua por decantación; el residuo solido se secó a $60^{\circ} \mathrm{C}$ durante 2 días hasta obtener una masa seca, la cual se trituró y se filtró por una malla.

\subsection{Métodos}

\subsubsection{Caracterización fisicoquímica del extracto de Capulín}

El pH del EC fue medido usando un $\mathrm{pH}$-metro portátil. El contenido de solidos solubles totales (TSS) se determinó mediante un refractómetro con rango de medición 0-90\% Brix. Para la acidez titulable (TA) se realizó una homogenización del FC en agua $(2: 10 \mathrm{w} / \mathrm{v})$, se 
filtró y se tomó una alícuota del sobrenadante, para posteriormente diluirla en agua (1:10 v/v) y finalmente hacer una titulación con $\mathrm{NaOH}(0.01 \mathrm{~N})$ y fenolftaleína como indicador; el porcentaje de TA se calculó en equivalentes de ácido gálico (GAE) de acuerdo a la Ec. 1 (Alia-Tejacal et al., 2002), donde $\mathrm{T}$ es volumen de $\mathrm{NaOH}$ en la titulación, $\mathrm{N}$ es la normalidad del $\mathrm{NaOH}, \mathrm{V}$ es el volumen del sobrenadante, $\mathrm{M}$ es volumen de la alícuota, $\mathrm{P}$ es masa de la muestra inicial, PM es el peso equivalente de ácido gálico (174.14) y F es factor de conversión (10).

$$
\mathrm{GAE}=\frac{\mathrm{T} * \mathrm{~N} * \mathrm{~V} * \mathrm{PM}}{\mathrm{M} * \mathrm{P} * \mathrm{~F}}
$$

El contenido de azúcares totales (TSC) se cuantifico con el método fenol-sulfúrico (Dubois et al., 1956), en el cual se utilizaron $0.5 \mathrm{~mL}$ de EC (1:7000 v/v), $0.5 \mathrm{~mL}$ de Fenol $5 \%$ y 2.5 $\mathrm{mL}$ de $\mathrm{H}_{2} \mathrm{SO}_{4}$ concentrado, y finalmente se realizaron lecturas a $490 \mathrm{~nm}$, expresando los resultados en equivalentes de glucosa (GE). El contenido total de polifenoles (TPC) se evaluó mediante método Folin-Ciocalteu (Özgen et al., 2010), diluyendo $1 \mathrm{~mL}$ del EC $(1: 20, \mathrm{v} / \mathrm{v})$ y adicionando $1.8 \mathrm{~mL}$ de carbonato sódico $7.5 \%$ y $0.6 \mathrm{~mL}$ de reactivo Folin $10 \%$, para finalmente realizar lecturas a $760 \mathrm{~nm}$ y expresar resultados en equivalentes de ácido gálico. La capacidad antioxidante (TEAC) fue medida mediante el método de estabilidad del radical DPPH• (Özgen et al., 2010), en el cual se utilizaron $0.1 \mathrm{~mL}$ de EC y $2.9 \mathrm{~mL}$ de DPPH• $0.1 \mathrm{mM}$, se realizaron lecturas a $515 \mathrm{~nm}$ utilizando al Trolox y al ácido ascórbico (AAE) como equivalentes.

\subsubsection{Microencapsulación}

El proceso de microencapsulación se llevó a cabo mediante secado por aspersión, operado el secador a una temperatura de entrada de $120^{\circ} \mathrm{C}$, una temperatura de salida de $79^{\circ} \mathrm{C}$, una aspiración de $35 \mathrm{~m}^{3} / \mathrm{h}$ y un flujo en la bomba de $3.915 \mathrm{~mL} / \mathrm{min}$. Para la formulación de dispersiones se realizó un diseño de experimentos (Tabla 3), donde se propusieron diferentes concentraciones de encapsulantes haciendo variaciones entre $1-10 \%$ de maltodextrina (MD) y de AC.

Tabla 3. Tratamientos (T) de la microencapsulación de FC con MD y AC.

\begin{tabular}{ccc}
\hline $\mathbf{T}$ & $\begin{array}{c}\mathbf{M D} \\
(\boldsymbol{\%})\end{array}$ & $\begin{array}{c}\mathbf{A C} \\
(\boldsymbol{\%})\end{array}$ \\
\hline 1 & 0 & 10 \\
2 & 0 & 10 \\
3 & 3 & 7 \\
4 & 5 & 5 \\
5 & 5 & 5 \\
6 & 7 & 3 \\
7 & 10 & 0 \\
8 & 10 & 0 \\
\hline
\end{tabular}




\subsubsection{Liberación y evaluación de componentes bioactivos}

Para la liberación de los compuestos bioactivos de los microencapsulados se utilizó la metodología modificada de Robert et al. (2010), para lo cual se diluyó el polvo en agua $(5: 1, \mathrm{w} / \mathrm{v})$ haciendo agitaciones vigorosas $(\mathrm{T}=3 \mathrm{~min})$, posteriormente se tomó una alícuota, se mezcló con etanol $(1: 2, \mathrm{v} / \mathrm{v})$ y se centrifugó $(13200 \mathrm{rpm}, 2 \mathrm{~min})$; el sobrenadante obtenido se mezcló nuevamente con etanol (1:2, v/v) y se centrifugó (13200 rpm, 2 min), finalmente se recuperó el sobrenadante con el contenido de compuestos bioactivos.

\subsubsection{Caracterización de los microencapsulados de EC}

El rendimiento del proceso se evaluó de acuerdo los componentes fenólicos retenidos en la superficie de los encapsulados, para lo cual se realizó una extracción Soxhlet (22.033 AOAC, 1960) de $3 \mathrm{~h}$, en la que se colocaron $5 \mathrm{~g}$ del polvo en un cartucho de celulosa y se utilizó éter etílico como disolvente. El porcentaje de rendimiento (R) se determinó con la Ec. 2, donde $\mathrm{Ci}$ es la concentración de compuestos fenólicos iniciales y Cs es la concentración de compuestos fenólicos superficiales.

$$
\mathrm{R}=\frac{\mathrm{Ci}-\mathrm{Cs}}{\mathrm{Ci}} * 100
$$

La actividad de agua ( $\mathrm{a}_{\mathrm{w}}$ ) se midió mediante un higrómetro. La humedad de los polvos fue evaluada por la técnica de calentamiento (modificado 10.064 AOAC, 1960) en la cual 100 $\mathrm{mg}$ de polvo microencapsulado se secaron en estufa a temperatura de $60^{\circ} \mathrm{C}$ durante 24 horas y el porcentaje de humedad $(\mathrm{H})$ se determinó por la $E c$. 3, donde MF es la masa de la muestra fresca y MS es la masa de la muestar seca.

$$
\mathrm{H}=\left(\frac{\mathrm{MF}-\mathrm{MS}}{\mathrm{MF}}\right) * 100
$$

La solubilidad $(\delta)$ de cada uno de los microencapsulados se determinó disolviendo el polvo en agua a $30^{\circ} \mathrm{C}(10: 1, \mathrm{w} / \mathrm{v})$, se centrifugó $(3000 \mathrm{rpm}, 5 \mathrm{~min})$ y $25 \mathrm{~mL}$ del sobrenadante se secaron en estufa $\left(105^{\circ} \mathrm{C}, 5 \mathrm{~h}\right)$ para posteriormente evaluar el porcentaje de solubilidad con la Ec. 4 (Shittu \& Lawal, 2007), donde SS es la masa del sobrenadante seco y M es la masa de la muestra inicial.

$$
\delta=\frac{\mathrm{SS}}{\mathrm{M}} * 100
$$

Las determinaciones de contenido total de polifenoles y de actividad antioxidante se evaluaron de la misma manera que para el EC. Y finalmente, se realizó una evaluación de la morfología y tamaño de los microencapsulados mediante microscopia electrónica de barrido (MEB), utilizando un recubrimiento de cobre para el análisis y realizando las lecturas con una ampliación de 2500x. 


\section{RESULTADOS}

\subsection{Caracterización fisicoquímica del EC}

En el Tabla 4 se muestra un $\mathrm{pH}$ ácido (4.56) en el extracto, un contenido de solidos solubles de 1.83 en la escala de ${ }^{\circ}$ Brix, $1.28 \%$ GAE de acidez titulable, un contenido de azúcares expresados en equivalente de glucosa de $44.20 \mathrm{mg} / \mathrm{L}$, un contenido de polifenoles de $199.87 \mathrm{mg} \mathrm{GAE} / \mathrm{mL}$ y un porcentaje de inhibición del radical $\mathrm{DPPH} \cdot$ de 45.06 , lo que equivale a una capacidad antioxidante de $140.01 \mathrm{mg} \mathrm{AAE} / \mathrm{L}$.

Tabla 4. Caracterización fisicoquímica del EC.

\begin{tabular}{lc}
\hline \multicolumn{1}{c}{ Determinación } & EC \\
\hline pH & $4.56 \pm 0.00$ \\
TSS $\left({ }^{\circ} \mathrm{Brix}\right)$ & $1.83 \pm 0.28$ \\
$\mathrm{TA}(\% \mathrm{GAE})$ & $1.28 \pm 0.02$ \\
$\mathrm{TSC}(\mathrm{mg} \mathrm{GE} / \mathrm{mL})$ & $44.20 \pm 6.20$ \\
$\mathrm{TPC}(\mathrm{mg} \mathrm{GAE} / \mathrm{mL})$ & $199.87 \pm 27.37$ \\
$\mathrm{TEAC} \mathrm{AAE}(\mathrm{mg} / \mathrm{mL})$ & $140.01 \pm 28.75$ \\
\%esultados expresados con la media & $45.06 \pm 9.09$ \\
\hline \multicolumn{2}{l}{ desviación estándar para cada muestra. }
\end{tabular}

\subsection{Rendimiento del proceso de microencapsulación}

El Tabla 5 muestra los resultados obtenidos para el rendimiento de recuperación de microencapsulado en todos los tratamientos ensayados. Se observa que el T8 (10-0\% MDAC, w/w) presentó el porcentaje más alto (89.13\%); mientras que el T1 (0-10\% MD-AC, w/w) obtuvo el menor rendimiento (28.99\%), lo cual indica que el rendimiento se ve afectado de forma positiva a medida que la concentración de MD se incrementa en la mezcla de agentes encapsulantes. 
Tabla 5. Rendimiento de los diferentes microencapsulados.

\begin{tabular}{cc}
\hline Tratamiento & Rendimiento $(\%)$ \\
\hline 1 & 28.99 \\
2 & 29.47 \\
3 & 31.01 \\
4 & 46.85 \\
5 & 30.29 \\
6 & 58.85 \\
7 & 87.83 \\
8 & 89.13 \\
\hline
\end{tabular}

\subsection{Caracterización de los microencapsulados}

El contenido de $\mathrm{H}$ en los tratamientos está en un intervalo de 1.78-4.49\%, la $\mathrm{a}_{\mathrm{w}}$ se encuentra entre $0.27-0.35$ y la $\delta$ va de 7.62 a $75.43 \%$. Además, los ocho tratamientos mostraron resultados de TPC entre $105.75-1370 \mathrm{mg}$ GAE/mL y un TEAC de $36.80-46.61 \%$ inhibición del DPPH• o 51.89-68.39 mg AAE/mL.

Tabla 6. Caracterización de los microencapsulados.

\begin{tabular}{|c|c|c|c|c|c|c|}
\hline \multirow[b]{2}{*}{$\mathbf{T}$} & \multirow[b]{2}{*}{$\mathbf{a}_{\mathbf{w}}$} & \multirow[b]{2}{*}{$\underset{(\%)}{\mathbf{H}}$} & \multirow[b]{2}{*}{$\begin{array}{c}\delta \\
(\%)\end{array}$} & \multirow{2}{*}{$\begin{array}{c}\text { TPC } \\
(\mathbf{m g} \\
\text { GAE/mL) }\end{array}$} & \multicolumn{2}{|c|}{ TEAC } \\
\hline & & & & & $\begin{array}{c}(\mathbf{m g} \\
\mathrm{AAE} / \mathbf{m L})\end{array}$ & \% DPPH• \\
\hline 1 & $0.29 \pm 0.00$ & $4.05 \pm 0.14$ & $7.62 \pm 0.37$ & $114.87 \pm 4.34$ & $60.79 \pm 4.58$ & $42.40 \pm 2.72$ \\
\hline 2 & $0.31 \pm 0.00$ & $3.98 \pm 0.12$ & $14.57 \pm 1.02$ & $118.52 \pm 8.55$ & $63.26 \pm 4.19$ & $43.56 \pm 2.49$ \\
\hline 3 & $0.29 \pm 0.00$ & $1.78 \pm 0.06$ & $55.92 \pm 0.86$ & $113.62 \pm 3.37$ & $68.39 \pm 3.66$ & $46.61 \pm 2.17$ \\
\hline 4 & $0.31 \pm 0.01$ & $4.49 \pm 0.15$ & $64.15 \pm 1.52$ & $137.00 \pm 1.51$ & $58.58 \pm 10.19$ & $40.77 \pm 6.06$ \\
\hline 5 & $0.34 \pm 0$ & & $52.57 \pm$ & $128.62 \pm 5.20$ & $61.13 \pm 6.43$ & 42.2 \\
\hline 6 & $0.28 \pm 0.00$ & $3.54 \pm 0.07$ & $15.30 \pm 0.29$ & $105.75 \pm 2.34$ & $54.67 \pm 8.90$ & $38.45 \pm 5.29$ \\
\hline 7 & $0.35 \pm 0.00$ & $2.66 \pm 0.20$ & $62.85 \pm 1.95$ & $120.62 \pm 1.88$ & $60.54 \pm 10.34$ & $41.94 \pm 3.82$ \\
\hline 8 & $0.27 \pm 0.00$ & $2.21 \pm 0.07$ & $75.43 \pm 0.15$ & $109.75 \pm 4.44$ & $51.89 \pm 4.98$ & $36.80 \pm 2.96$ \\
\hline
\end{tabular}

Resultados expresados con la media $(n=3) \pm$ la desviación estándar para cada muestra.

La morfología de los polvos se muestra en la Fig. 1, en donde se puede ver que los microencapsulados con un solo agente encapsulante ( $(\mathrm{y}$ y b) tienen tamaños homogéneos entre sí $(1-10 \mu \mathrm{m})$, mientras que los polvos tratados con la mezcla de encapsulantes (c y d) presentan tamaños diversos. 

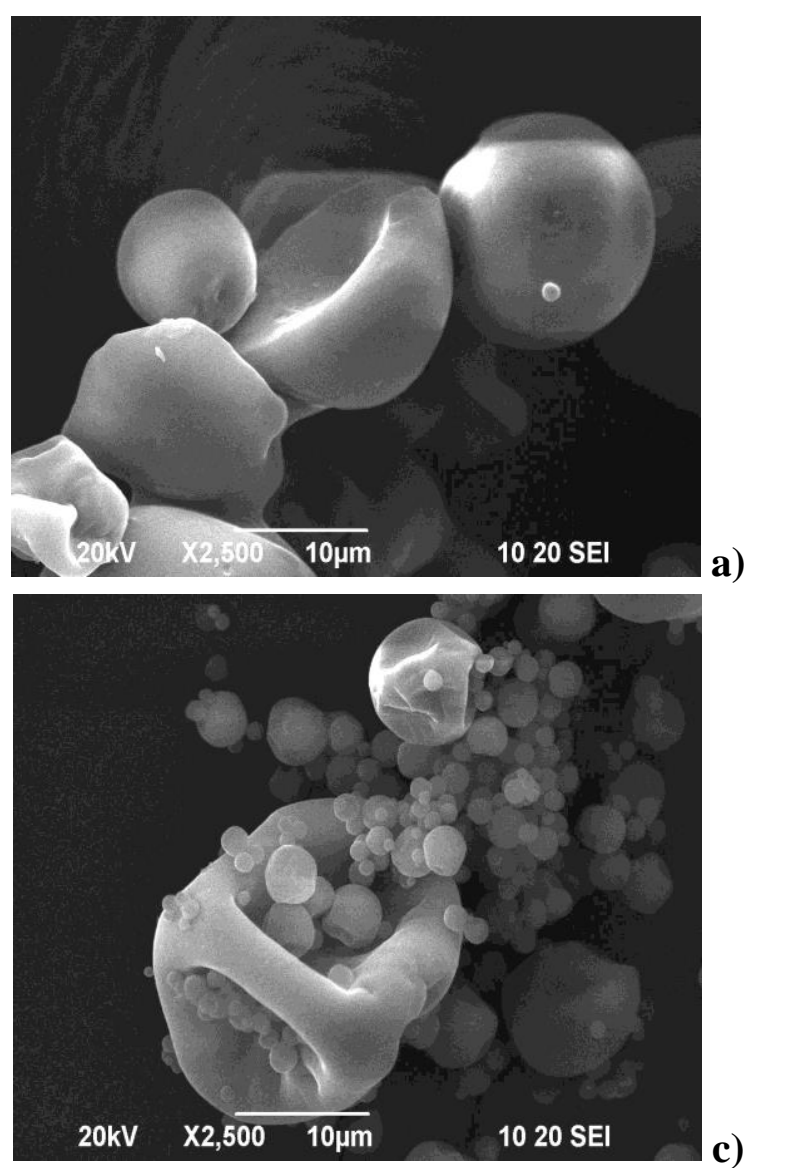

a)

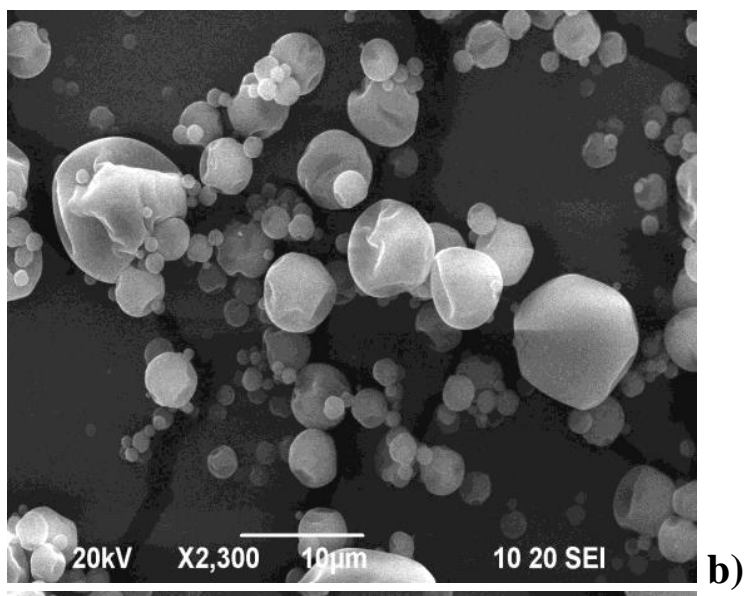

c)

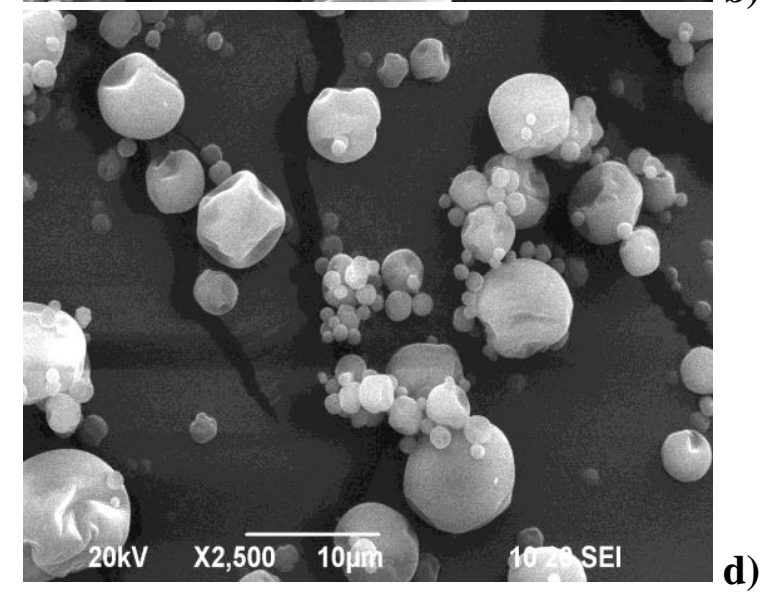

Fig. 1. Micrografías de los microencapsulados visualizadas mediante MEB a 2500x: a, T1 (10\% AC); b, T8 (10\% MD); c, T3 (3-7\% MD-AC); y d, T6 (7-3\% MD-AC).

\section{DISCUSIÓN}

Aunque el análisis fisicoquímico realizado al EC sirvió como partida para cuantificar algunos componentes presentes al inicio del proceso, los valores reportados Jiménez et al. (2011) en cuanto al contenido fenólico y a la capacidad antioxidante de un extracto acuoso de Capulín son superiores (746 mg GAE/mL y $51.38 \%$, respectivamente) a lo encontrado en este estudio; sin embargo, estos componentes dependen directamente del estado de madurez y de las condiciones de cultivo del fruto como el tipo de riego, la temperatura y la geografía y ubicación del suelo (Kader \& Barret, 2005). Por otro lado, la caracterización del extracto, permitió la visualización del deterioro de los componentes bioactivos durante el tratamiento de secado por aspersión, mismos que están relacionados con factores ambientales y por el manejo mismo del extracto (luz, T, aireación); de manera que permitió la evaluación del proceso al final de la investigación.

Por otro lado, todos los polvos microencapsulados son considerados microbiológicamente estables por que presentan valores de actividad de agua inferiores a 0.6 (Quek et al., 2007) y una humedad menor a 5\% (Ebadat, 2013); además, la solubilidad es un parámetro de importancia para la liberación del material activo, por lo que el T8 fue el polvo mayormente 
soluble (Castro-Muñoz et al., 2015) y con un mayor contenido de polifenoles. Sin importar la disminución de los componentes fenólicos en los encapsulados, la cual está asociada a la oxidación por las temperaturas de operación del secador (Ferrari et al., 2012), la capacidad antioxidante de éstos no se vio afectada, debido a que los compuestos fenólicos antes de degradarse modifican su estructura para formar polímeros que no pierden la actividad biológica inmediatamente (Mishra et al., 2014).

En cuanto a la morfología de los microencapsulados, la forma colapsada de las partículas en las cuatro micrografías se debe al proceso de secado, pues la rápida eliminación de agua en la cámara del secador genera arrugas y depresiones en la superficie de los polvos (Rosenberg et al, 1985), por lo que los microencapsulados obtenidos están clasificados como películas y no como esferas (Walton, 2000).

\section{CONCLUSIONES}

El secado por aspersión resulto ser una buena alternativa para la microencapsulación de componentes bioactivos extraídos del fruto del Capulín, ya que no afecto las propiedades antioxidantes del fruto; además, permitió obtener polvos estables microbiológicamente por su baja actividad de agua y su mínimo contenido de humedad.

Por otro lado, los tratamientos con maltodextrina mejoraron la capacidad de atrapamiento del contenido total de polifenoles, permitiendo la recuperación hasta del $70 \%$ los componentes; mientras que los tratamientos que incluían almidón de chinchayote presentaron mejores resultados en la conservación de la actividad antioxidante del extracto del fruto de Capulín.

\section{AGRADECIMIENTOS}

Los autores agradecen al IPN (20170153) por el financiamiento otorgado.

\section{CONFLICTO DE INTERESES}

Los autores declaran que no existe conflicto de intereses.

\section{REFERENCIAS}

A. O. A. C. 1960. Official Methods of Analysis of the Association of Official Agricultural Chemists (Methods of Analysis A. O. A. C). $9^{\circ}$ edition.

Alia-Tejacal I., Colinas-León M. T., Martínez-Damián M. T. \& Soto-Hernández M. R. 2002. Factores fisiológicos, bioquímicos y de calidad en frutos de zapote mamey (Pouteria sapota Jacq. H.E. Moore \& Stearn) durante poscosecha. Revista Chapingo Serie Horticultura. 8(2): 263-281.

Anal A. K. \& Singh H. 2007. Recent advances in microencapsulation of probiotics for industrial applications and targeted delivery. Trends in Food Science \& Technology. 18: 240-251. 
BDMTM, 2009. Biblioteca Digital de la Medicina Tradicional de México. Consultada el 22 de noviembre de $\quad 2017$ de http://www.medicinatradicionalmexicana.unam.mx/monografia.php?l=3\&t=Capul\%C3\%A $\underline{\text { Dn\&id }=7046}$

Belloso O. M., Fortuny R. S. \& Oliu G. O. 2009. Cambios microbiológicos, físicoquímicos y sensoriales de la fruta fresca cortada, de origen templado. Pp. 44-63. En González Aguilar G. A., Álvarez Parrilla E., de la Rosa L., Olivas I. G., \& Ayala Zavala J. F. 2009. Aspectos nutricionales y sensoriales de vegetales frescos cortados. México, D. F.: Trillas.

Biessels H. W., van der Kerk-van Hoof A. C., Kettenes-van den Bosch J. J. K. \& Salemink C. A. 1974. Triterpenes of Prunus serótina and P lusitánica. Phytochemistry. 13: 203-207.

Castro-Muñoz R; Barragán-Huerta B. \& Yáñez-Fernández J. 2015. Use of gelatinmaltodextrin composite as an ancapsulation support for clarified juice from purple cactus pear (Opuntia stricta). LWT-Food Science Technology. 62: 242-248.

Deladino L., Anbinder P. S., Navarro A. S. \& Matino M. N. 2008. Encapsulation of natural antioxidants extracted from Ilex paraguariensis. Carbohydrates Polymers. 71: 126-134.

Desai K. G. H \& Park H. J. 2005. Recent developments in microencapsulation of food ingredients. Drying Technology: An International Journal. 23(7): 1361-1394.

Dubois M., Gilles K. A., Hamlton J. K., Rebers P. A. \& Smith F. 1956. Colorimetric method for determination of sugars and related substances. Analytical Chemistry. 28(3): 350-356.

Ebadat V. 2013. Ensuring process safety in food powder production: the risk of dust explosion. Pp. 260-282. En Bhandari B., Bansal N., Zhang M. \& Schuck P. Handbook of food powders. Processes and properties. New Delhi, India: Woodhead Publishing Limited.

Fang Z. \& Bhandari B. 2010. Encapsulation of polyphenols - a review. Trends in Food Science \& Technology. 21: 510-523.

Ferrari C. C., Marconi Germer S. P. \& de Aguirre J. M. 2012. Effects of stray-drying conditions on the physicochemical properties of blackberry powder. Drying Technology: An International Journal. 30(2): 154-163.

Ganga Z. N. \& Corke H. 1999. Physical properties of starch of Asian-adapted potato varieties. Journal of the Science of Food and Agriculture. 79: 1642-1646.

García-Aguilar L., Rojas-Molina A., Ibarra-Alvarado C., Rojas-Molina J. I., VázquezLandaverde P. A., Luna-Vázquez F. J. \& Zavala-Sánchez M. A. 2015. Nutritional Value and Volatile Compounds of Black Cherry (Prunus serotina) Seeds. Molecules. 20: 34793495 . 
Gharsallaoui A., Roudaut G., Chambin O., Voilley A. \& Saurel R. 2007. Applications of spray-drying in microencapsulation of food ingredients: An overview. Food Research International. 40: 1107-1121.

Jimenez M., Castillo I., Azuara E. \& Beristain C. I. 2011. Antioxidant and antimicrobial activity of capulin (Prunus serótina subsp capuli) extracts. Revista Mexicana de Ingeniería Química. 10(1): 29-37.

Kader A. A. \& Barret D. M. 2005. Classification. Composition of Fruits, and Postharvest Maintenance of Quality. Pp. 3-22. En Barrett D. M., Somogyi, L. \& Ramaswamy H. Processing fruits: science and technology, second edition. Florida: CRC Press LLC.

Mishra P., Mishra S. \& Mahanta C. L. 2014. Effect of maltodextrin concentration and inlet temperature during spray drying on physicochemical and antioxidant properties of amla (Emblica officinalis) juice powder. Food and Bioproducts Processing. 92: 252-258.

Ordaz-Galindo A., Wesche-Ebeling P., Wrolstad R. E., Rodriguez-Saona L., \& ArgaizJamet A. 1999. Purification and identification of Capulin (Prunus serotina Ehrh) anthocyanins. Food Chemistry. 65: 201-206.

Özgen M., Scheerens J. C., Reese R. N. \& Miller R. A. 2010. Total phenolic, anthocyanin contents and antioxidant capacity of selected elderberry (Sambucus Canadensis L.) accessions. Pharmacognosy Magazine. 6(23): 198-204.

Pennington J. A. T. 2002. Food composition databases for bioactive food components. Journal of Food Composition Analysis. 15: 419-434.

Phisut N. 2012. Spray drying technique of fruit juice powder: some factors influencing the properties of product. International Food Research Journal. 19(4): 1297-1306.

Porras-Saavedra J., Palacios-González E., Yáñez-Fernández J., Mazzobre M. F., Buera M. P. \& Alamilla-Beltrán L. 2015. Caking process and microstructural changes of Wall materials used in spray-drying process. Pp. 629-636. En Gutiérrez-López G. F., AlamillaBeltrán L., Buera M. P., Welti-Chanes J., Parada-Arias E. \& Barbosa-Cánovas G. V. Water Stress in Biological, Chemical, Pharmaceuitical and Food Systems. New York: Springer Science+Business.

Quek S.Y., Chok N.K., \& Swedlund P. 2007. The physochemical properties of spray-dried watermelon powders. Chemical Engineering Processing. 46: 386-392.

Robert P., Gorena T., Romero N., Sepulveda E., Chavez J. \& Saenz C. 2010. Encapsulation of polyphenols and anthocyanins from pomegranate (Punica granatum) by spray drying. International Journal of Food Science and Technology. 45: 1386-1394.

Rosenberg M., Kopelman I. J. \& Talmon Y. 1985. A scanning electron microscopy study of microencapsulation. Journal of Food Science. 50: 139-144. 
Shahidi F. \& Han X-Q. Encapsulation of Food Ingredientes. 1993. Critical Reviews in Food Science and Nutrition. 33(6): 501-547.

Shittu T. A. \& Lawal M. O. 2007. Factors affecting instant properties of powdered cocoa beverages. Food Chemistry. 100: 91-98.

Walton D. E. 2000. The morphology of spray-dried particles a qualitative view. Drying Technology: An International Journal. 18(9): 1943-1986.

Yáñez Fernández J., Salazar Montoya J. A., Chaires Martínez L., Jiménez Hernández J., Márquez Robles M. \& Ramos Ramírez E. G. 2002. Aplicaciones biotecnológicas de la microencapsulación. Avance y Perspectiva. 21: 313-319. 